\title{
Determination of the bilinear stress-crack opening curve for normal- and high-strength concrete
}

\author{
A. M. FATHY , B. SANZ, J. M. SANCHO and J. PLANAS
}

Departamento de Ciencia de Materiales, E.T.S. de Ingenieros de Caminos, Canales y Puertos, Universidad Politecnica de Madrid, C/Profesor Aranguren s/n, 28040 Madrid, Spain, Faculty of Engineering, Ain Shams University, Abdo Basha, Cairo, Egypt, Universidad CEU-San Pablo, Escuela Politecnica Superior, Campus de Monteprincipe, Boadilla del Monte, 28668 Madrid, Spain

Received in final form 7 April 2008

ABSTRACT An improved version of the method proposed to ACI committee 446 and to RILEM TC 187-SOC to determine the fracture parameters of concrete is applied in this study to several mixtures of normal and high-strength concretes. The results are processed with a $\mathrm{C}++$ program developed by the authors to automatise the mathematical operations required to obtain the bilinear softening curve of concrete from the experimental results. Numerical simulations of the tests are also carried out using finite elements with an embedded cohesive crack. The comparison between numerical and experimental results confirms that the experimental and numerical procedures are appropiate for normalstrength concretes and high-strength concretes.

Keywords cohesive crack; concrete; finite elements; fracture; high-strength concrete.

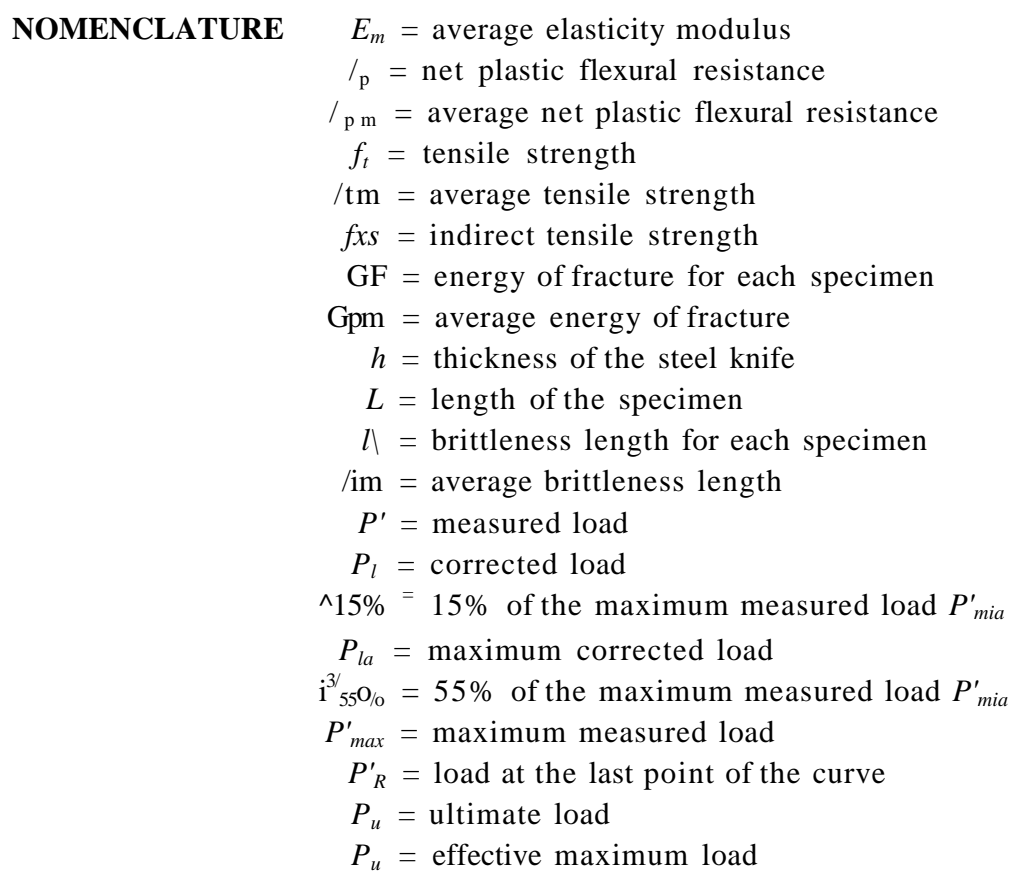




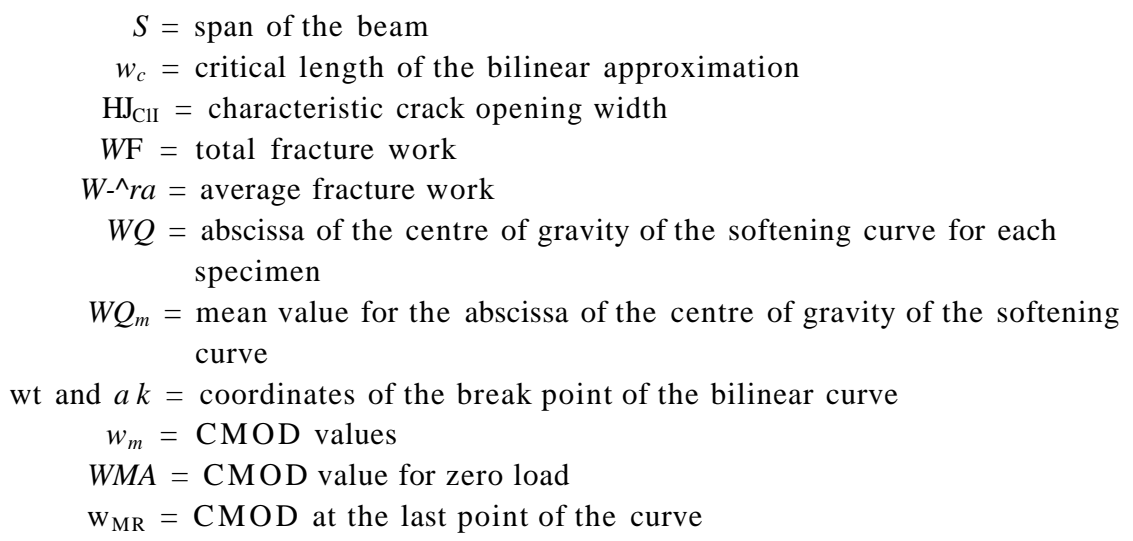

\section{INTRODUCTION}

The model introduced by Hilleborg et al. in 1976 to describe concrete progressive cracking as the fictitius crack and currently known as the cohesive crack model is able to describe the fracture of stressed concrete with a good precision/complexity ratio.

The main input for the model is the softening curve, which presents a noticeable nonlinearity. Various simplified analytical expressions have been proposed for the softening curve to make the mathematical formulation easier. One of them is the bilinear curve, which has been used since the 1980s and which we will refer to in this work.

This article presents an updated implementation of the proposed experimental method described which determines the four parameters necessary to define the bilinear softening curve of concrete from the combination of the results obtained from Brazilian tests and three-point bending tests. A program in $\mathrm{C}++$ has been designed to automatize the mathematical operations. It automatically computes the necessary parameters to define the curve, directly from the experimental data files, without any intervention of the user other than selecting the data files and introducing the basic geometrical data. Such automation provides an increased traceability and reliability of the data reduction procedure.

Little information exists in the literature about the ability of tests such as those analyzed in the paper to perform smoothly when applied to high-strength concrete, which is of concern as the increased brittleness and load capacity of the specimens might introduce instabilities in the experimental system. Thus, one of the objectives of the present work is to assess the ability of the experimental method to deal, without any special tuning, both with ordinary and with high-strength concrete.

A numerical analysis by means of finite elements with embedded cohesive crack is carried out in order to ver- ify that the treatment given to the experimental data is appropriate. The behaviour of the beams is numerically predicted from the stress-crack opening curve obtained from the experimental data, which allowed us to confirm that the numerical method, which was known to be appropriate for normal-strength concrete, is also valid for high-strength concrete.

\section{PROPOSED METHOD}

\section{Tests procedures}

The tests to determine the softening curve of concrete are based on the combination of the results obtained after carrying out diametral cylinder splitting tests (Brazilian tests, for short) and stable three-point bending tests on notched beams.

Brazilian tests are carried out on standard cylindrical specimens $150 \mathrm{~mm}$ in diameter and $300 \mathrm{~mm}$ in length, according to the standard ASTM C496, with a few improvements dictated by previous research, such as narrower load-bearing strips and limited rate of loading.

In the bending tests, beams of rectangular cross section with a central notch are tested with a central load and a loading span equal to three times the depth of the beam. The tests are carried out under crack opening control (CMOD) to ensure stability. Weight compensation is used so that the test can be controlled until the end of the test without the specimen unstably falling under its own weight.

The CMOD, the load and the mid-span deflection are measured during the test. The deflection is determined relative to a reference frame, which is held on points on the upper face of the specimen on the vertical of the supports, so that the measurement does not include inelastic deformation originating in the supports.

In this work, a beam of 500 x $100 \times 100 \mathrm{~mm}$ and with a central notch $33 \mathrm{~mm}$ in depth has been used (see Fig. 1). The weight compensation is obtained by means 


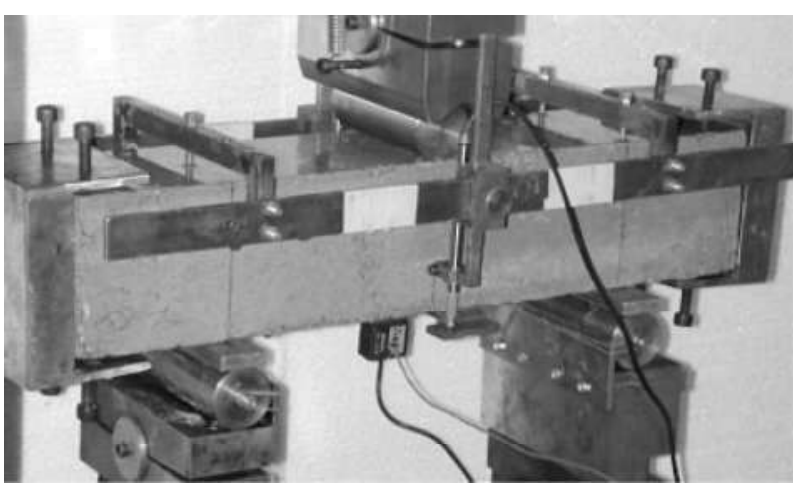

Fig. 1 View of the experimental arrangement for the three-point bending tests.

of two steel counter weights clamped at the ends of the beam. Deflection is measured with two inductive displacement transducers. To measure the crack opening width, a clip-on gauge is placed between two steel plates that are fastened to the beam at both sides of the crack with screws. One of these plates is wide enough to protrude out of the beam on each side so providing a reference plane for the displacement transducers. The beam is supported by cylinders over two rigid steel plates, one of which can rotate around an axis normal to the loading plane to minimize the torsion.

During the preparation and realization of the test, the beam must be kept permanently wet. The measuring instruments are calibrated and mounted on the test specimen; next, a preload between $5 \%$ and $10 \%$ of the estimated maximum load is applied. Then, the machine control is switched to CMOD and the test started, with an initial rate selected (from a trial test) so that the maximum load is reached between 3 and $5 \mathrm{~min}$. Once the load reaches the maximum and decreases one third of it, the rate is progressively increased to keep a constant unloading rate until the end of the test, which is conventionally reached when the CMOD equals $4 / 3$ of the beam depth (1.333 $\mathrm{mm}$ in this case).

\section{Data reduction to determine the bilinear softening curve}

A scheme of the bilinear curve and the parameters that define it is shown in Fig. 2. The method used to determine the bilinear approximation is fully explicit, and the principles supporting it are described in detail elsewhere. Suffice it to say, here, that the tensile strength $f_{t}$ is estimated from the Brazilian tests, and the remaining parameters from the records of the Load $(\mathrm{P})$ versus CMOD and displacement (8) of the bending tests: the elastic modulus $E$ from the initial slope of the $P$ -

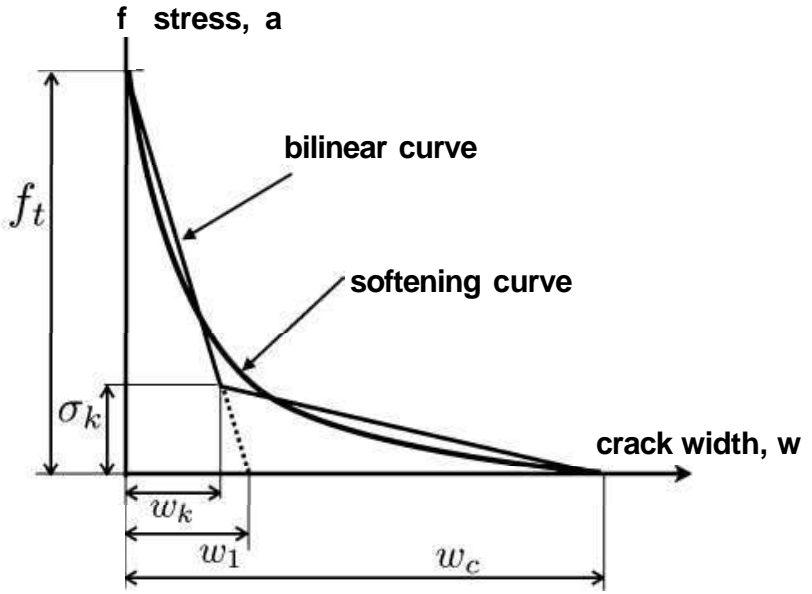

Fig. 2 Scheme of the bilinear softening curve of concrete.

CMOD curve, the horizontal intercept of the initial softening segment $(w)$ from the peak load, the fracture energy Gp from the area under the $P-8$ curve and the centroid of the softening curve from the far-tail behaviour. From these basic values, all the remaining parameters are determined from simple geometrical relations. The detailed steps and equations necessary to reduce the experimental data are listed in Appendix 1.

Although conceptually simple, processing the data requires a large number of operations that, if carried out by hand, make it difficult to trace and to debug. Therefore, there was a desire for a tool to carry out the operations automatically. In order for the tool to be multiplatform, portable and free, it has been coded in $\mathrm{C}++$ using objectoriented programing.

The necessary information the program needs to work are the geometric data of each specimen: width $B$, depth $\mathrm{D}$, loading span $S$ and notch depth $a o$, and the path to the files with the records of data obtained during the test. The input of data is facilitated by means of a graphic user interface.

\section{EXPERIMENTAL RESULTS}

Four types of concrete were prepared: two normalstrength concretes (NSC), with design strength of 25 and $40 \mathrm{MPa}$, and two high-strength concretes (HSC) with design strength of 80 and $90 \mathrm{MPa}$. For each type of concrete, the following specimens were made: six cylindrical specimens (three for compression tests and three for the Brazilian tests) and six beams for three-point bending tests. The dimensions of the specimens are those described in the section 'Test procedures'.

The tests were stable until the end, independently of the concrete type; no special tuning whatsoever was required 
Table 1 Experimental results

\begin{tabular}{lllll}
\hline \multirow{2}{*}{ Series } & \multicolumn{3}{c}{ NSC } & \multicolumn{3}{l}{ HSC } \\
\cline { 2 - 5 } & 1 & 2 & 3 & 4 \\
\hline$f_{c}(\mathrm{MPa})$ & 28.7 & 39.1 & 85.2 & 87.7 \\
$f t(\mathrm{MPa})$ & 2.24 & 2.84 & 5.82 & 6.18 \\
$E(\mathrm{GPa})$ & 31.6 & 32.5 & 45.1 & 47.7 \\
$w_{c}(\mathrm{~mm})$ & 0.272 & 0.293 & 0.519 & 0.863 \\
$\mathrm{G}_{\mathrm{F}}(\mathrm{N} / \mathrm{m})$ & 68.5 & 86.3 & 121 & 126 \\
$\mathrm{a}_{\mathrm{k}}(\mathrm{MPa})$ & 0.375 & 0.378 & 0.125 & 0.042 \\
$w_{k}(\mid \mathrm{im})$ & 15.6 & 21.8 & 30.6 & 34.9 \\
$/ \mathrm{i}(\mathrm{mm})$ & 133 & 144 & 121 & 136 \\
$I_{\mathrm{ch}}(\mathrm{mm})$ & 432 & 349 & 162 & 158 \\
\hline
\end{tabular}

$f_{c}=$ compression strength; $f_{t}=$ tensile strength; $E=$ elastic modulus; $w_{c}=$ critical crack opening; Gp = fracture energy; $\left(w^{\wedge}, a^{\wedge}\right)=$ coordinates of the kink point of the bilinear curve; $\Lambda=E w \bigvee 2 f_{t}=$ brittleness length; $l_{c}^{\wedge}=E G\{j \AA ⿻$ characteristic length.

for HSC specimens. In this respect we can conclude that the experimental setup is robust enough to deal with HSC.

The results are shown in Table 1 . The actual compression strengths are 28.7 and $39.1 \mathrm{MPa}$ for the two NSC mixes, and 85.2 and 87.7 for the HSC mixes. It can be observed that/t, $E_{m}$ and $\mathrm{Gp}_{\mathrm{m}}$ increase when the compression strength increases, whereas / $\mathrm{c}$ decreases and the brittleness length $\lambda$ is almost constant. The relation $a^{\wedge} / f_{t}$ is low for the NSC (between 0.13 and 0.17 ) and very low for high-strength concrete (between 0.021 and 0.0068).

The load-CMOD curves obtained in each test are grouped according to the type of concrete and are shown in Figs. 3-6.

\section{NUMERICAL SIMULATION AND DISCUSSION}

The tests have been numerically simulated taking as the input the experimental bilinear softening curves using the finite element program continuum oriented finite element (COFE) ), which incorporates a special finite element developed : Such elements are based on describing the crack as a displacement discontinuity (strong discontinuity kinematics). In order to avoid the necessity of tracking algorithms, the element implements an original approach, which is based on the combination of a cohesive crack with central forces (the traction vector is proportional to the crack displacement vector), and a limited adaptability of the crack, which can reorientate perpendicular to the maximum principal stress direction while its opening is small (less than about $0.2 \mathrm{Gp} / /_{\mathrm{t}}$ ); this prevents crack locking while keeping the formulation strictly local.

Further simulations have been conducted using a highly accurate boundary integral method known as the smeared

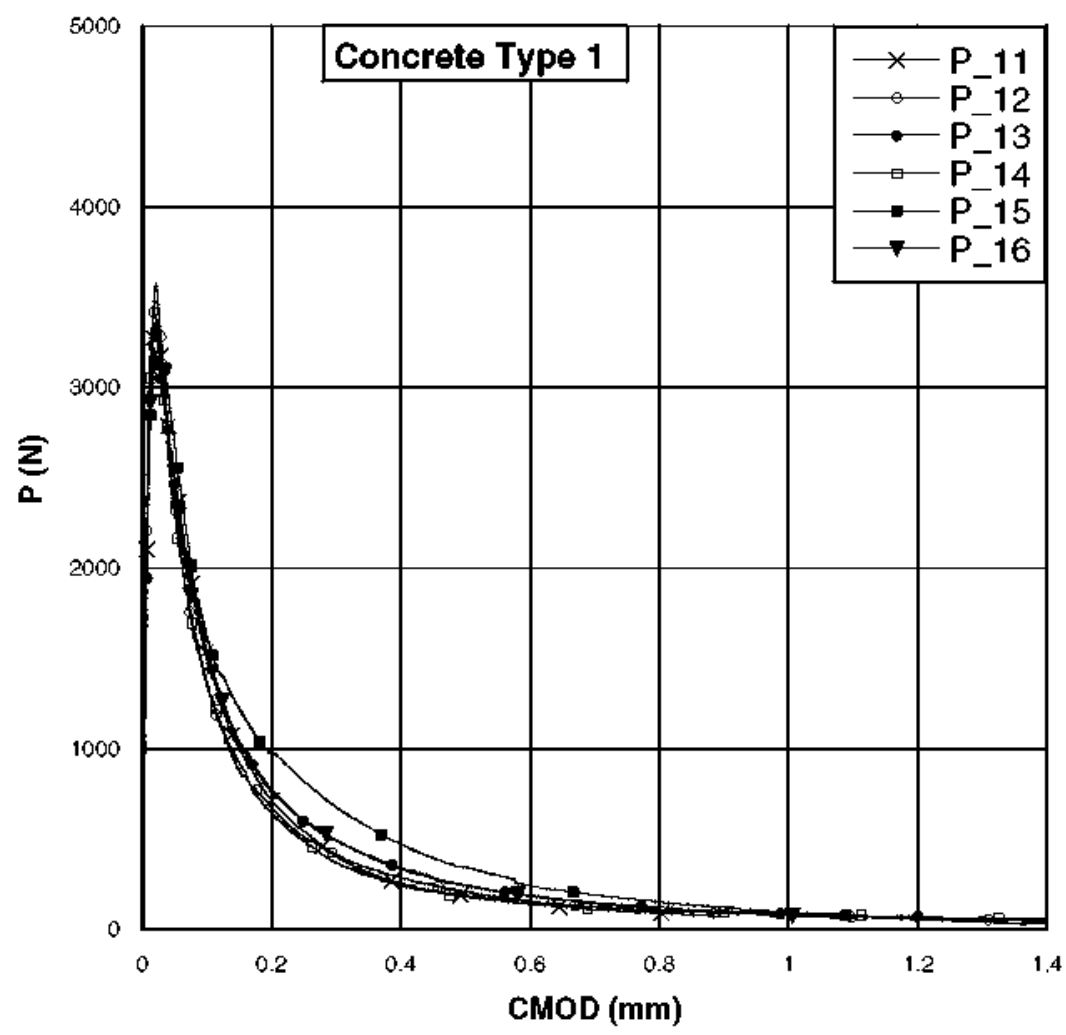

Fig. 3 Experimental Load - CMOD curves for normal-strength concrete. Type 1 . 
Fig. 4 Experimental Load - CMOD curves for normal-strength concrete. Type 2 .

Fig. 5 Experimental Load - CMOD curves for high-strength concrete. Type 3 .
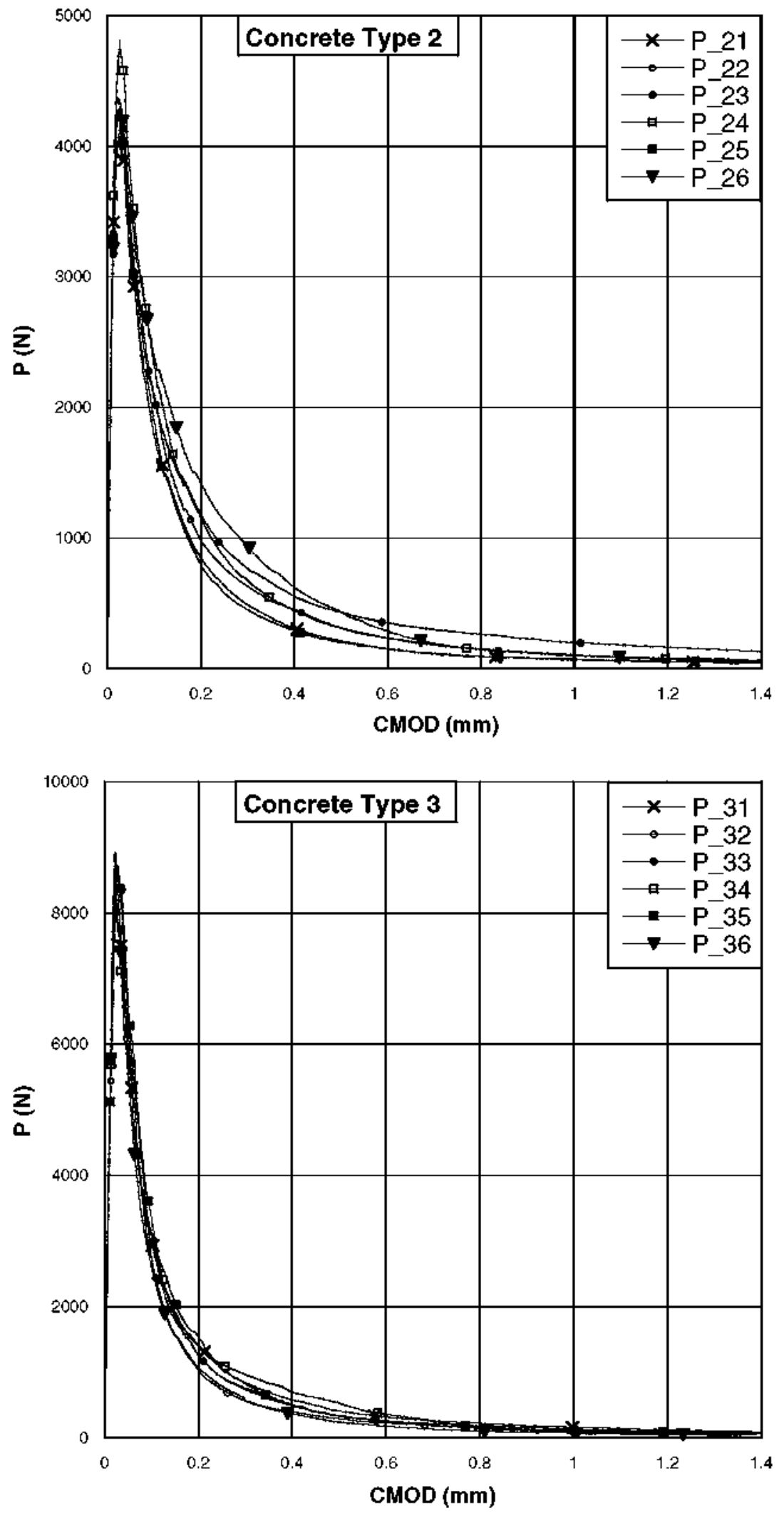

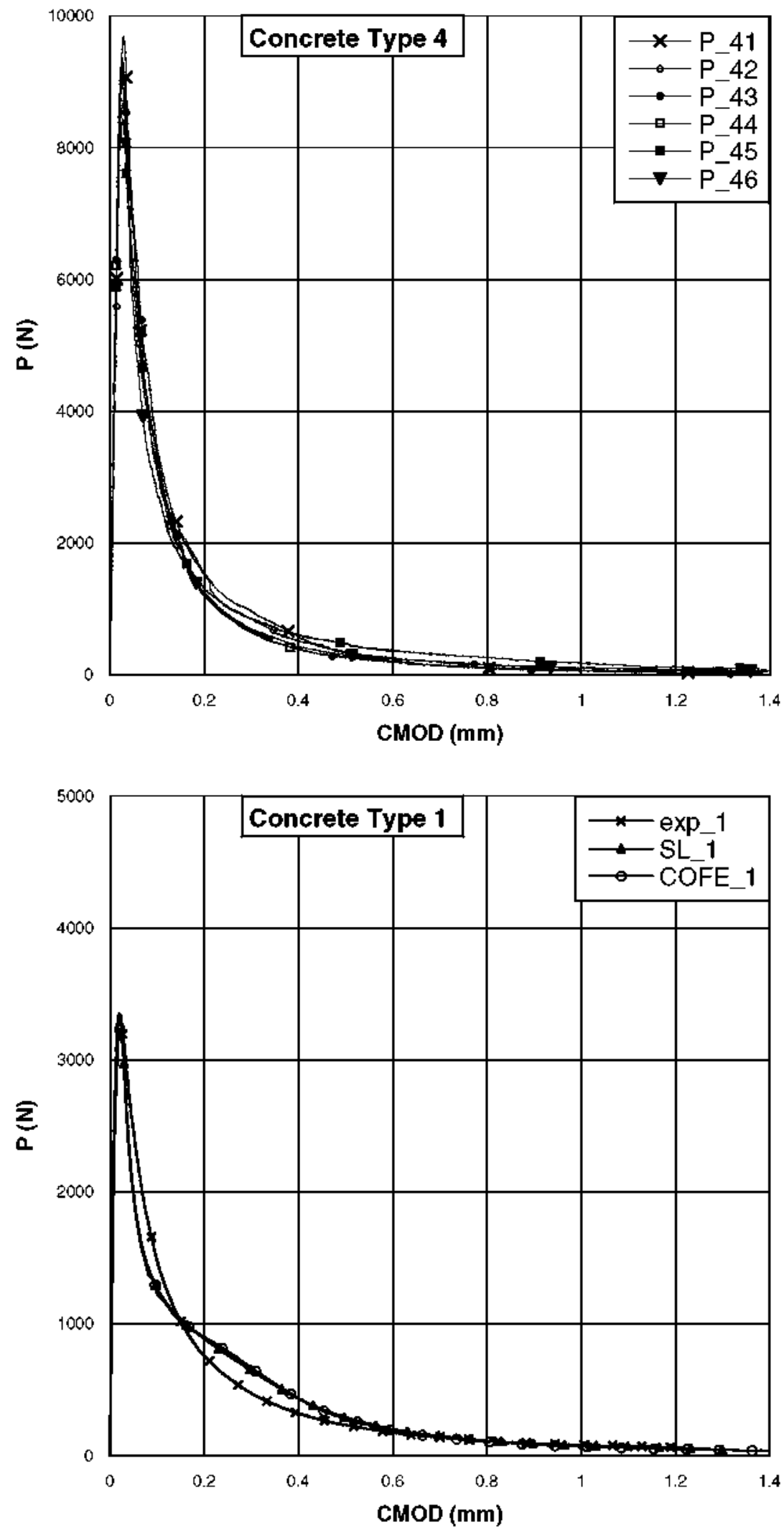

Fig. 6 Experimental Load - CMOD curves for high-strength concrete. Type 4.
Fig. 7 Comparison between experimental and numerical Load - CMOD curves simulated with SplittingLab and COFE for normal-strength concrete, series 1. 
Fig. 8 Comparison between experimental and numerical Load - CMOD curves simulated with SplittingLab and COFE for normal- strength concrete, series 2.

Fig. 9 Comparison between experimental and numerical Load - CMOD curves simulated with SplittingLab and COFE for high-strength concrete, series 3.
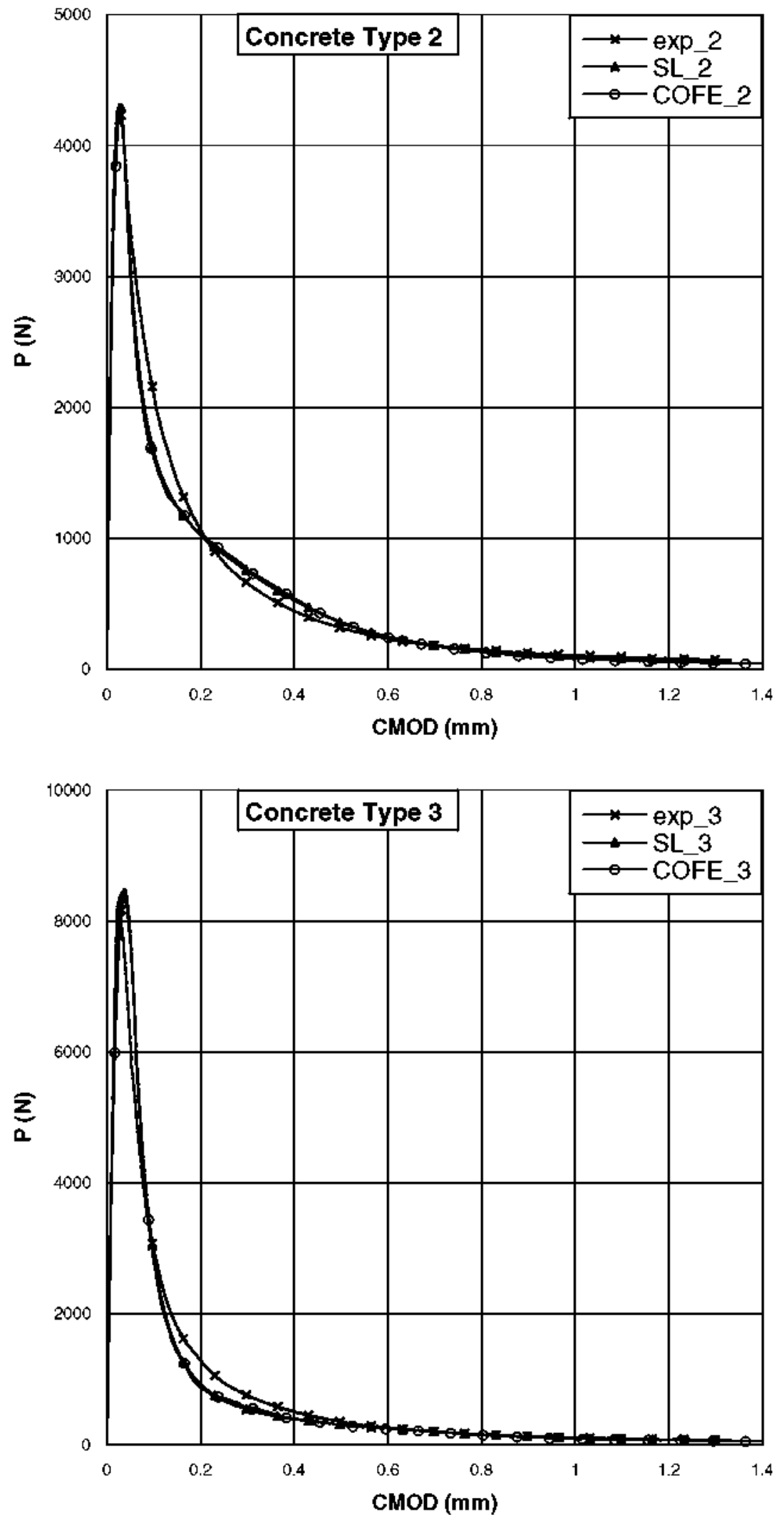


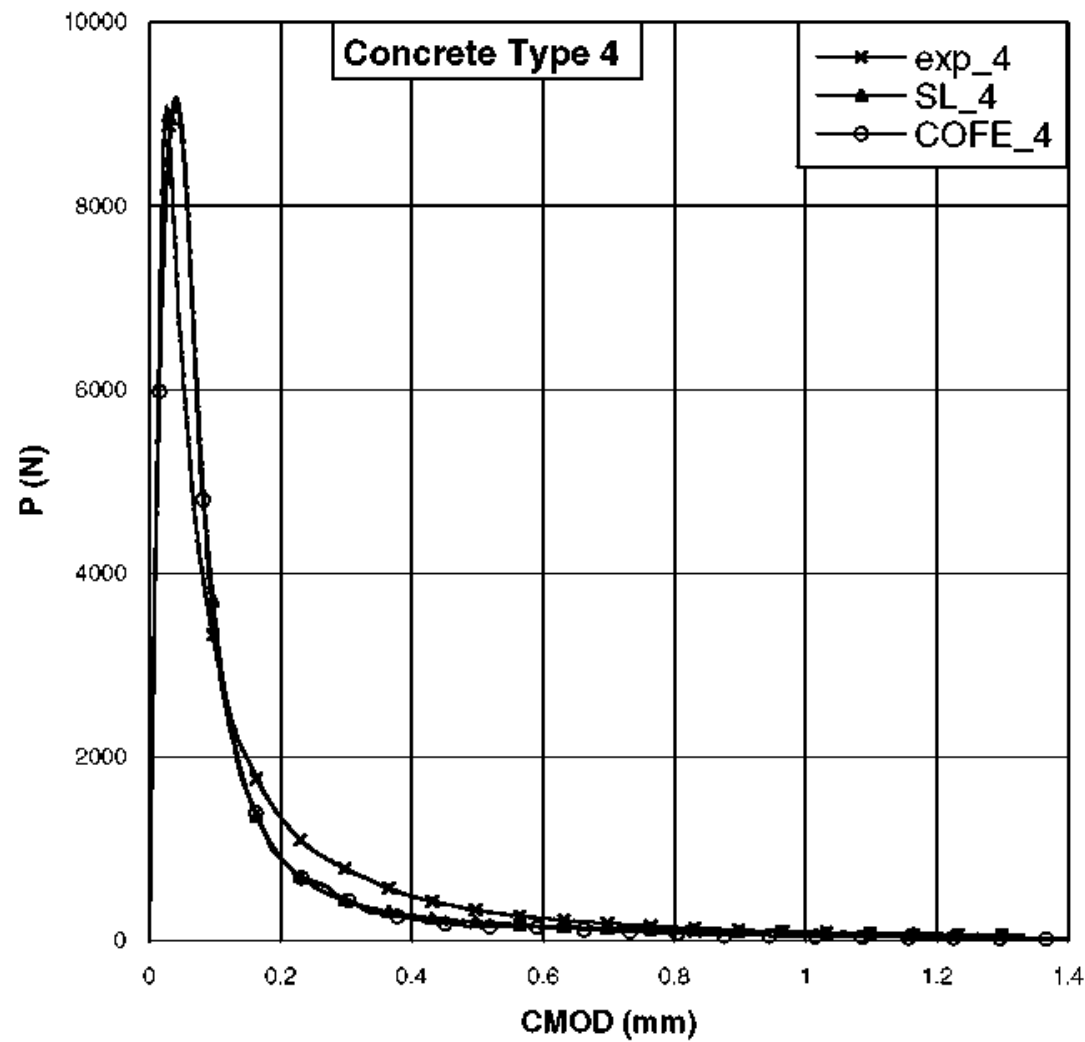

Fig. 10 Comparison between experimental and numerical Load - CMOD curves simulated with SplittingLab and COFE for high-strength concrete, series 4 . tip superposition method implemented in the SplittingLab program, developed by the fourth author

In Figs. 7, 8, 9 and 10 the load-CMOD curves obtained from experimental results and from numerical simulations are compared for each group of concrete. Each experimental curve is the average of the corresponding curves to all the specimens of the same type of concrete. From the comparison, we can appreciate that the simulations catch very well the initial slope, the peak load, the total area of the curve and the far-tail of the curve; this is so, because the data reduction method is designed so as to fit these features as closely as possible. The deviations seen in the post-peak are no doubt due to the fact that the bilinear approximation is a relatively rough approximation of the actual softening, which is smooth and does not show any kind of cusp. In summary, the method provides the best approach one can expect for a bilinear approximation of a softening curve, both for normal and high-strength concrete. One may also note that the two numerical predictions (FEM with embedded adaptable crack and boundary integral method) give essentially identical results.

\section{CONCLUSIONS}

The main conclusion reached in this work is that both the experimental method and the numerical approximation based on finite elements with embedded adaptable cohe- sive crack are robust enough to deal with $\mathrm{HSC}$ as well as with NSC.

A secondary conclusion is that, as expected, the mechanical properties (stiffness, strength, toughness) and the traditional brittleness (inverse of the characteristic length /eh) are larger for HSC than for NSC, while the brittleness length, the inverse of which measures the brittleness around the peak, is roughly constant for the materials tested in this work.

\section{Acknowledgements}

The authors want to acknowledge financial support received from the Spanish National Education Department through the grant BIA2005-09250-C03-01. The present work was conducted in the framework of the projects DUMELNPA sponsored by the Consejeria de Educacion de la Comunidad Autonoma de Madrid, and SEDUREC integrated in the program CONSOLIDER-LNGENIO 2010 .

\section{REFERENCES}

Hilleborg, A., Modeer, M. and Petersson, P. E. (1976) Analysis or crack formation and crack growth in concrete by means of fracture mechanics and finite elements. Cement Concrete Res. 6, 773-782.

Bazant, Z. P. and Planas, J. (1998) Fracture and Size Effect in Concrete and Other Quasibrittle Materials. CRC Press, Boca Raton, FL. 
Elices, M., Guinea, G. V., Gomez, F.J. and Planas, J. (2002) The cohesive zone model: advantages, limitations and challenges. Eng. Frac. Mech. 69, 137-163.

Planas, J., Elices, M., Guinea, G. V., Gomez, F. J., Cendon, D. A. and Arbilla, I. (2003) Generalizations and specializations of cohesive crack models. Eng. Frac. Mech. 70 , 1759-1776. Guinea, G. V, Planas, J. and Elices, M. (1994) A general bilinear fitting for the softening curve of concrete. Mat. Struct. 27, 99-105.

Planas, J., Guinea, G. V. and Elices, M. (1999) Size effect and inverse analysis in concrete fracture. Int. J. Fract. 95, 367-378. Planas, J., Guinea, G. V. and Elices, M. (2005) Standard test method for bilinear stress-crack opening curve of concrete. Proposal submitted to ACI Committee 446 (2002), revised (2005).

SanchoJ. M., Planas J., Fathy A. M., Galvez, J. C. and Cendon, D. A. (2007) Three-dimensional simulation of concrete fracture using embedded crack elements without enforcing crack path continuity. Int. J. Numer. Analytic. Methods Geomechan. John Wiley, 31, 173-187, February.

Rocco, C, Guinea, G. V., Planas, J. and Elices, M. (1999) Mechanisms of rupture in the splitting test. ACIMater. J. 96, 52-60.

Rocco, C, Guinea, G. V., Planas, J. and Elices, M. (1999) Size effect and boundary conditions in the Brazilian test: experimental verification. Mat Struct. 32, 10-217. RILEM (1985) Determination of the fracture energy of mortar and concrete by means of three-point bend tests on notched beams. Mat. Struct. 18, 285-290. (RILEM Draft Recommendation, TC 50-FMC Fracture Mechanics of Concrete).

Elices, M., Guinea, G. V. and Planas, J. (1997) On the measurement of concrete fracture energy using three point bend tests. Mat. Struct. 30, 375-376.

Planas, J. and Sancho, J. M. (2007) Instructions to use COFE (Continuum Oriented Finite Elements. Departamento de Ciencia de Materiales. Universidad Politecnica de Madrid, Spain.

\section{APPENDIX 1: Detailed data reduction procedure}

a. Compute the average tensile strength $f_{l m}$ from the Brazilian tests as follows:

a. 1. Compute the tensile strength for each specimen $\mathrm{as} / \mathrm{t}=/_{\mathrm{a}}=2 P_{U} /(J T D L)$, where $\left./ \mathrm{t}\right) /_{\mathrm{a}}, \quad \mathrm{P}_{\mathrm{u}}, \quad D$ and $L$ are, respectively, the tensile strength, indirect tensile strength, ultimate load, diameter and length of the specimen.

a.2. Compute/an as the mean of all the individual $f_{t}$ values.

b. From the bending tests, determine the mean elastic modulus $E_{m}$ as follows:

b.1. On the raising branch of the $\mathrm{P}^{\prime}-\mathrm{CMOD}$ curve, select the test points whose measured load $P$ lies between 15 and $55 \%$ of the maximum load.

b.2. Fit a straight line to the previous set of points using a linear regression of CMOD versus $P$, the slope of which is taken as the initial com- pliance of the specimen $\mathrm{C} ;=A w_{m} / A P$, where $w_{m}$ denotes the CMOD value.

b.3. Compute the elastic modulus of the specimen as $E=6 S a_{0} V_{l}\left(a_{Q}^{\prime}\right) /\left(C_{i} B D^{2}\right)$, where $S, B$ and $D$ are the span, width and depth of the beam, \#o is the notch depth, $a_{0}^{\prime}=(\# \mathrm{O}+h) /(D+h)$, where $h$ is the thickness of the steel knife used to clamp the CMOD gauge and

$$
\begin{aligned}
& V_{x}(a)=0.8-1.7 \mathrm{a}+2.4 \mathrm{a}^{2}+-\wedge \mathrm{r} \mathrm{y} \\
& (1-\mathrm{a})^{2} \\
& +\stackrel{4 D}{\wedge} \mathrm{r}\left(-0.04-0.58 \mathrm{a}+1.47 \mathrm{a}^{2}-2.04 \mathrm{a}^{3}\right)
\end{aligned}
$$

b.4. Compute $E_{m}$ as the mean of the individual values.

c. For each bending tests, determine the constant $A$ characterizing the far-tail asymptote as follows:

c.1. Eliminate from the record the points with CMOD > 4D/300.

c.2. Record the values of the CMOD and the load at the last point of the curve (H?MR and $\mathrm{P}^{\wedge}$, say).

c.3. For each point in the record, calculate the corrected load as $P i=P-\mathrm{P}^{\wedge}$.

c.4. Draw the curve of Pi vs. CMOD and determine the CMOD value for zero load on the raising branch, call it H?MA-

c.5. For all the points of the tail such that $\mathrm{Pi}<$ $0.05 P \backslash_{u}$, where $\mathrm{Pi}_{\mathrm{u}}$ is the maximum corrected load, compute the auxiliary values

$$
\begin{gathered}
X=(W / S)^{2}\left[l /\left(w_{M}-w_{M A}\right)^{2}-l /\right. \\
\text { OMR - WMA } \left.)^{2}\right]
\end{gathered}
$$

c.6. Plot the values of $\mathrm{Pi}$ versus $\mathrm{X}$ and perform a curve fitto the equationPi $=X(A+K X)$. Record the resulting value $o £ A$ to three significant digits.

d. For each bending test, determine the net plastic flexural strength $f_{p}$ as follows:

d.1. For each specimen, calculate the effective maximum load as $\mathrm{P}_{\mathrm{u}}=\mathrm{P}_{1 \mathrm{u}}+A /\left(W M R-\mathrm{J}^{\prime \prime M A}\right)^{2}$

d.2. For each specimen, calculate the net plastic flexural strength as $/_{\mathrm{p}}=P_{U} S /\left[2 B\left(D-a_{0}\right)^{2}\right]$.

e. For the bending tests, determine the mean energy of fracture $\mathrm{Gp}_{\mathrm{m}}$ as follows:

e.1. For each specimen, plot he corrected load Pi versus the deflection $S$ and determine the deflections $5 \mathrm{~A}$ and $5 \mathrm{R}$ (in the loading and unloading parts, respectively) corresponding to zero load.

e.2. For each specimen, compute the measured fracture work as the area enclosed by the curve $\mathrm{Pi}-S$ and the $S$ axis.

e.3. Compute the total fracture work as $W-\%=$ $W_{F m}+2 A /\left(S_{R}-5_{\mathrm{A}}\right)$. 
e.4. Compute the energy of fracture for each specimen as $\mathrm{G}_{\mathrm{F}}=W_{F} /\left[B\left(D-\ll_{0}\right)\right]$.

e.5. Compute $\mathrm{Gp}_{\mathrm{m}}$ as the mean of the individual values.

f. Compute the mean value of the abscissa of the centre of gravity of the softening curve WGm as the mean of the individual values computed for each specimen as $z v_{G}=4 A /\left(B S G_{F}\right)$

g. Determine the parameters of the bilinear approximation of the softening curve as follows:

g.1. Compute the brittleness length for each specimen as

$l_{1}=\left(1-\alpha_{0}^{m}\right) D\left[\frac{c_{1}}{\left(x^{2}-1\right)^{2}}+\frac{c_{2}}{x^{2}}\right]$,

where $x=f_{t m} / f_{p}$, and $m, c \backslash$ and $c j$ are constants which depend on the span-to-depth ratio $(S / D)$; for $S / D=3, m=1.7, c_{x}=11.2$ and $c_{2}=2.365$. g.1. Compute $\Lambda_{m}$ as the mean of the individual values h-

g.2. Compute the parameter $w \backslash$ for the concrete as wi $=2 f_{t m} l i_{m} / E_{m}$

g.3. Compute the characteristic crack opening $w_{c} h$ as $\mathrm{W}_{\mathrm{c}} \mathrm{h}=\mathrm{GFm} / / \mathrm{tm}$

g.4. Compute the critical length of the bilinear approximation as

$$
\begin{aligned}
& w_{c}=w_{\mathrm{ch}} \frac{3 w_{\mathrm{Gm}}-w_{1}}{2 w_{\mathrm{ch}}-w_{1}} \\
& \times\left[1+\sqrt{1-\frac{2 w_{1}\left(3 w_{\mathrm{Gm}}-2 w_{\mathrm{ch}}\right)\left(2 w_{\mathrm{ch}}-w_{1}\right)}{\left.w_{\mathrm{ch}}\left(3 w_{\mathrm{Gm}}-w_{1}\right)\right)^{2}}}\right]
\end{aligned}
$$

g. Compute the coordinates $(<7 \mathrm{t}, \mathrm{wt})$ of the kink point of the bilinear curve as:

$$
\sigma_{\mathrm{k}}=f_{\mathrm{tm}} \frac{2 w_{\mathrm{ch}}-w_{1}}{w_{\mathrm{c}}-w_{1}} \text { and } w_{\mathrm{k}}=w_{1} \frac{w_{\mathrm{c}}-2 w_{\mathrm{ch}}}{w_{\mathrm{c}}-w_{1}}
$$

